\title{
Positive surgical margin rates during the robot-assisted laparoscopic radical prostatectomy learning curve of an experienced laparoscopic surgeon
}

Anthony F. Adili, MBBCH; Julia Di Giovanni, MBBCH; Emma Kolesar; Nathan C. Wong MD; Jen Hoogenes; Shawn Dason, MD; Bobby Shayegan, MD, FRCSC

Division of Urology, McMaster University, Hamilton, ON, Canada

Cite as: Can Urol Assoc J 2017; Epub ahead of print. http://dx.doi.org/10.5489/cuaj.4588

Published online November 1, 2017

$* * *$

Abstract

Introduction: Since its introduction, robot-assisted laparoscopic radical prostatectomy (RARP) has gained widespread popularity, but is associated with a variable learning curve. Herein, we report the positive surgical margin (PSM) rates during the RARP learning curve of a single surgeon with significant previous laparoscopic radical prostatectomy (LRP) experience.

Methods: We performed a prospective cohort study of the first 400 men with prostate cancer treated with RARP by a single surgeon (BS) with significant LRP experience. Our primary outcome was the impact of case timing in the learning curve on margin status. Our analysis was conducted by dividing the case numbers into quartiles (Q1-Q4) and determining if a case falling into an earlier quartile had an impact on margin status relative to the most recent quartile (Q4). Results: The Q1 cases had an odds ratio for margin positivity of 1.74 compared to Q4 ( $\mathrm{p}=0.1$ ). Multivariate logistic regression did not demonstrate case number to be a significant predictor of PSM. The mean Q1 operative time was 207.4 minutes, decreasing to 179.2 by Q4 $(\mathrm{p}<0.0001)$. The mean Q1 estimated blood loss was $255.1 \mathrm{ml}$, decreasing to 213.6 by Q4 ( $\mathrm{p}=0.0064)$. There was no change in length of hospitalization within the study period.

Conclusions: Even when controlling for copredictors, a statistically significant learning curve for PSM rate of a surgeon with significant previous LRP experience was not detected during the first 400 RARP cases. We hypothesize that previous LRP experience may reduce the RARP PSM learning curve. 


\section{Introduction}

Robot-assisted laparoscopic radical prostatectomy (RARP) has been widely adopted over the last decade. Estimates from the United States suggest that $67 \%$ of patients undergoing radical prostatectomy had RARP in 2010, compared to only 8\% in 2004. ${ }^{1}$ The adoption of any novel surgical techniques is associated with a learning curve that may affect surgical quality. With progression along the RARP learning curve, improvements may be seen in various domains including operative time, estimated blood loss, positive surgical margin rate, urethrovesical anastomosis time, complications, length of hospital stay, transfusion rate, early continence, potency and conversion rate. ${ }^{2}$

Positive surgical margin (PSM) rates after RARP range from 6.5-32\% and serve as an important marker of surgical quality. ${ }^{3}$ A PSM after RARP is independently associated with an increased risk of biochemical recurrence. ${ }^{3}$ Patients with a PSM after RARP will also be more likely to receive adjuvant or salvage radiation therapy. ${ }^{4}$ An extremely variable learning curve has been documented for PSM rates after RARP — with prior series estimating a plateau in PSM rates after 20 to 1600 cases. $^{2}$ It is likely that many factors, including prior experience with non-robotic or pure laparoscopic radical prostatectomy (LRP), may impact the RARP learning curve. In this study, we report the PSM rates during the RARP learning curve of a single surgeon with significant previous LRP experience.

\section{Methods}

\section{Study design}

Participants were enrolled in this study if they underwent RARP at St. Joseph's Healthcare Hamilton (SJHH) by a single surgeon (BS). Consecutive participants were enrolled in this study from March 2012 to April 2015 after institutional review board approval was obtained. This was a cohort study in which outcomes were prospectively collected. Participants were excluded if they had an aborted procedure, had a conversion to an open surgical approach, were undergoing salvage RARP for radio-recurrent prostate cancer, had suspected stage T4 disease preoperatively, had received a course of neoadjuvant androgen deprivation therapy, had no malignancy found on the RARP specimen, or were undergoing cytoreductive RARP for oligometastatic disease.

\section{Perioperative management}

Participants were diagnosed with prostate cancer by transrectal ultrasound-guided prostatic biopsy. Participants received a preoperative anesthetic consultation and basic laboratory panel. Participants on anticoagulation had a preoperative thrombosis consultation and were bridged as necessary. Participants were permitted to be on low-dose antiplatelets (aspirin 81mg) if felt necessary by thrombosis physicians. Participants with high-risk disease (PSA $>20 \mathrm{ng} / \mathrm{ml}$ or Gleason 8-10) were staged with a bone-scan and computed tomographic (CT) scan of the abdomen and pelvis. Participants with suspected locally-advanced disease based on rectal examination or CT scan also underwent preoperative magnetic resonance imaging (MRI). The postoperative course after RARP followed a standardized institutional clinical pathway. Participants generally did not have a drain placed and went home with a urethral catheter on 
postoperative day 1 .

\section{Operative technique}

All RARP procedures were performed by a single surgeon (BS) using the da Vinci Si surgical system (Intuitive Surgical Inc., Sunnyvale, CA, USA). RARP was performed via a transperitoneal approach. Our RARP dissection begins with a limited posterior dissection to identify the seminal vesicles and vasa deferentia, followed sequentially by release of the bladder and development of the retropubic space, bilateral obturator fossa lymphadenectomy, resection of preprostatic fat and nodal tissue, endopelvic fascial incision, bladder neck dissection, an antegrade approach to pedicle division and nerve sparing, ligation and division of the dorsal venous complex, division of the prostatic apex and urethra, specimen removal, and vesicourethral anastomosis. No significant changes in the RARP technique were made during the study period. Because patients with a low risk of lymph node metastases generally undergo active surveillance at our center, bilateral obturator fossa lymphadenectomy is routinely performed and submitted with preprostatic nodal tissue for nodal staging. Participants had intrafascial nerve sparing when possible, with partial (interfascial or extrafascial) ipsilateral neurovascular bundle sparing for cases of high volume and/or high grade disease. Wide neurovascular bundle excision was performed for cases of ipsilateral high volume and/or high grade disease with suspected extracapsular extension based on clinical stage, visual inspection, tissue reaction, or MRI findings. Trainees are involved as bedside assistants for the RARP procedure and perform certain steps of the RARP procedure on a trainee console. Despite this, all the dissection during steps relevant to margin status (apical dissection, intrafascial nerve sparing, bladder neck in extensive tumors) was performed by the operating surgeon (BS). Prior to performing RARP, the operating surgeon (BS) had performed 600 LRP procedures in independent practice. The technique employed during LRP was transperitoneal and followed a similar approach as that of the RARP procedure. Criteria for preoperative staging, lymphadenectomy, and nerve-sparing were the same for LRP as RARP.

\section{Pathologic details}

Staging was performed according to the $7^{\text {th }}$ edition of the American Joint Committee on Cancer (AJCC) staging system. ${ }^{5}$ A dedicated genitourinary pathologist analyzed each specimen for margin status. Margin positivity was analyzed according to the International Society of Urological Pathology (ISUP) Working Group 5 Consensus. ${ }^{6}$ Margins were considered positive if there was tumor located at the inked prostatic margin. The location of margin positivity was recorded. Although comments were made on the extent of margin positivity in certain cases, this was not routinely quantified. Whole-mount sectioning was not performed.

\section{Statistical analysis}

The primary outcome of this study was the impact of case timing in the learning curve on margin status. This analysis was conducted by dividing the case numbers into quartiles (Q1-Q4) and determining if a case falling into an earlier quartile had an impact on margin status relative to the most recent quartile (Q4). This was assessed with an odds ratio with the most recent quartile 
(Q4) used as a reference. Logistic regression of case number as a continuous variable predictor of margin status was also performed. Co-predictors utilized in the logistic regression model were derived from previous series and included T3 vs. T2 stage, BMI, prostate weight, and PSA. ${ }^{7}$ Secondary outcomes included the impact of case quartile on operative time, estimated blood loss and length of hospital stay. Subgroup analysis was conducted to determine the effect of quartile on T2 and T3 PSM rates. Statistical analysis was performed with Medcalc Version 14.8 (Medcalc Software). Odds ratios and their statistical significance were computed in the methods previously described by Altman ${ }^{8}$ and Sheskin ${ }^{9}$ respectively. Student's t-testing was used to compare mean values where appropriate. Differences were considered statistically significant when the two-tailed $\mathrm{P}$ value was less than 0.05 . Where appropriate, the most recent quartile was used as the reference standard, with statistical testing performed in relation to this.

\section{Results}

A total of 400 participants met study inclusion criteria. No participants met exclusion criteria, and so this study was an analysis of the first 400 RARP cases performed by the operating surgeon (Table 1). The mean time elapsed during each quartile was 306.8 days, during which a mean of 2.6 cases were performed each week. Median patient age was 63.8 and median PSA was $6.9 \mathrm{ng} / \mathrm{ml}$.

Overall mean operative duration was 187.2 minutes and mean estimated blood loss was $240.9 \mathrm{ml}$ (Table 2). Bilateral nerve sparing was performed in 241/400 patients (60.3\%). A total of 157/400 (39\%) patients had pT3 disease and 39/400 (9.8\%) had Gleason 8-10 disease on final pathology (Table 3). There were 82 positive margins, of which 53 (64.6\%) arose from those with pT3 disease and 29 (35.4\%) from those with pT2 disease. Of the 82 positive margins, 43 (52.4\%) were positive apical margins.

The first quartile of cases had an odds ratio for margin positivity of 1.74 (95\% CI 0.90 3.36, $\mathrm{P}=0.1$ ) relative to the most recent quartile (Table 4). The odds ratio for pT2 positive margins was 1.69 ( $95 \% \mathrm{CI} 0.62-4.58, \mathrm{P}=0.30$ ). The odds ratio for $\mathrm{pT} 3$ positive margins was 1.45 (0.62-3.41, $\mathrm{P}=0.39$ ). When looking at the second or third quartiles relative to the most recent quartile, no significant differences were noted. Multivariate logistic regression did not demonstrate case number to be a significant predictor of PSM (Exp[B] 0.998 95\% CI 0.9941.002, $\mathrm{P}=0.329$ ).

The first quartile operative time was a mean of 207.4 minutes, decreasing to 179.2 by quartile $4(\mathrm{P}<0.0001)$. The first quartile estimated blood loss was a mean of $255.1 \mathrm{ml}$ and this decreased to $213.6 \mathrm{ml}$ by quartile $4(\mathrm{P}=0.0064)$. There was no change in length of hospitalization within the study period. Again, there were no differences noted in operative time, or estimated blood loss when quartiles 2 and 3 were compared to quartile 4 .

\section{Discussion}

In this series of 400 consecutive RARP performed by an experienced LRP surgeon, no learning curve was detected for PSM rates in all cases or in subgroup analysis of pT2 cases or pT3 cases. The learning curve in adopting RARP for surgeons with significant LRP experience is not well 
documented. Our literature search has uncovered only a limited number of learning curve series of surgeons with explicitly stated LRP experience (Table 5). Several other authors have also reported that they did not detect a learning curve in PSM rates during their initial experience with RARP, although this was not universal. Comparing these series directly proves difficult due to differing methodology - some authors compare their early RARP outcomes to their most recent LRP outcomes while others divide their RARP outcomes into groups based on case number.

Abboudi et al. ${ }^{2}$ have previous suggested in their systematic review that RARP may have a PSM learning curve ranging from 20-1000 or more cases. The generalizability of this finding to our study is limited as this is based primarily on data from surgeons that had limited laparoscopic experience or limited to no experience with LRP. While RARP and LRP differ in their technical approach, these operations are fundamentally similar in their conceptual and anatomic approach. We hypothesize that this similarity may hasten the RARP learning curve and explain the difficulty in detecting a significant PSM learning curve when adopting RARP after significant LRP experience. Alternate hypotheses as to why a learning curve was not detected in PSM rates during this study including the fact that the learning curve likely occurs within the first 100 cases, subtle differences over time are not reflected statistically, the operating surgeon is still early in the learning curve with gains occurring later after more than 400 cases, or that more challenging cases were pursued with time that were not reflected by the reported clinicopathologic parameters. Ultimately further follow-up and perpetual analysis of our outcomes will be necessary to determine whether the PSM rate has actually reached a plateau.

In contrast to our findings with PSM rates, we did detect a reduction in EBL and OR time with increasing case quartile. These findings are consistent with most previous studies reporting a reduction in operative time and blood loss with increasing experience (Table 5). ${ }^{2}$ While these measures do not necessarily reflect surgical quality, they do reflect improved efficiency resulting from familiarity with the procedure and technology.

Limitations of this study include the use of PSM rates as a primary outcome, which is only a surrogate of a clinically meaningful oncologic outcome - cancer-specific mortality (CSM). A PSM after RARP has been shown to be independently associated with an increased risk of biochemical recurrence and patients with a PSM after RARP will also be more likely to receive adjuvant or salvage radiation therapy. ${ }^{3-4}$ While relevant, the translation of these findings into CSM is unclear.

Additionally, our prospective data collection did not include certain data that would have contributed to this study. Study outcomes (PSM rate, EBL and OR time) were not being collected during the LRP era, which may have served as a control group. Finally, functional outcomes were not assessed in this study. Previous studies have suggested that improvements in functional outcomes occur during the RARP learning curve. ${ }^{2}$ Given the high survival rates of many patients with localized prostate cancer regardless of margin status, these are important clinical outcomes. Additionally, we could have considered other confounders on the learning curve which have been previously described such as days between cases. ${ }^{17}$ 
The study of the RARP learning curve is important because of its relevance in credentialing robotic surgeons. In their recent review, Lee et al. have advocated for an increased emphasis on proficiency with basic robotic skills and procedural tasks rather than number of completed cases in robotic surgery credentialing. ${ }^{18}$ This fits intuitively with a learning curve whose extent and method of measurement are uncertain. In keeping with this, Lovegrove et al. have validated a RARP Assessment Score to assess competency at high-risk steps of RARP. ${ }^{19}$ The RARP Assessment Score divides the procedure into 41 steps, which are then rated by a mentor on each case from 0 to 5 . These authors did demonstrate a learning curve in multiple steps and found that the study participants rated the assessment tool acceptable, feasible, and having educational impact. This tool, however, is limited by its uncertain translation to relevant oncologic or functional outcomes.

Ultimately, credentialing a RARP surgeon will need to integrate an assessment of technical skill, oncologic outcomes, functional outcomes, and efficiency-related outcomes. Proficiency will need to be judged based on outcomes, not solely based on surgical volume. Understanding factors that affect the learning curve, such as prior LRP experience, will be important in establishing credentialing standards.

\section{Conclusion}

Even when controlling for co-predictors, a statistically significant learning curve for positive surgical margin (PSM) rate of a surgeon with significant previous LRP experience with was not detected during robot-assisted radical prostatectomy (RARP) in this prospective cohort study. We hypothesize that previous laparoscopic radical prostatectomy experience may reduce the RARP PSM learning curve. 


\section{References}

1. Lowrance WT, Eastham JA, Savage C, et al. Contemporary open and robotic radical prostatectomy practice patterns among urologists in the United States. The Journal of Urology. 2012;187:2087-2093.

2. Abboudi H, Khan MS, Guru KA, et al. Learning curves for urological procedures: a systematic review. BJU international. 2014;114:617-629.

3. Yossepowitch O, Briganti A, Eastham JA, et al. Positive surgical margins after radical prostatectomy: a systematic review and contemporary update. European Urology. 2014;65:303-313.

4. Thompson IM, Valicenti RK, Albertsen P, et al. Adjuvant and salvage radiotherapy after prostatectomy: AUA/ASTRO Guideline. The Journal of Urology. 2013;190:441-449.

5. Edge SB, American Joint Committee on Cancer., American Cancer Society. AJCC cancer staging handbook : from the AJCC cancer staging manual. 7th ed. New York: Springer; 2010.

6. Tan PH, Cheng L, Srigley JR, et al. International Society of Urological Pathology (ISUP) consensus conference on handling and staging of radical prostatectomy specimens. Working group 5: surgical margins. Modern Pathology. 2011;24:48-57.

7. Patel VR, Coelho RF, Rocco B, et al. Positive surgical margins after robotic assisted radical prostatectomy: a multi-institutional study. The Journal of urology. 2011;186:511517.

8. Altman DG. Practical statistics for medical research: CRC press; 1990.

9. Sheskin DJ. Handbook of parametric and nonparametric statistical procedures: crc Press; 2003.

10. Jaffe J, Castellucci S, Cathelineau X, et al. Robot-assisted laparoscopic prostatectomy: a single-institutions learning curve. Urology. 2009;73:127-133.

11. Wolanski P, Chabert C, Jones L, Mullavey T, Walsh S, Gianduzzo T. Preliminary results of robot-assisted laparoscopic radical prostatectomy (RALP) after fellowship training and experience in laparoscopic radical prostatectomy (LRP). BJU international. 2012;110:6470.

12. Barret E, Sanchez-Salas R, Secin F, et al. Robotic prostatectomy positive margins learning curve. Journal of Endourology. 25; 2011:A62-A63.

13. Stolzenburg J-U, Qazi HA, Holze S, et al. Evaluating the learning curve of experienced laparoscopic surgeons in robot-assisted radical prostatectomy. Journal of Endourology. 2013;27:80-85.

14. Di Pierro GB, Wirth JG, Ferrari M, Danuser H, Mattei A. Impact of a single-surgeon learning curve on complications, positioning injuries, and renal function in patients undergoing robot-assisted radical prostatectomy and extended pelvic lymph node dissection. Urology. 2014;84:1106-1111. 
15. Ku JY, Ha HK. Learning curve of robot-assisted laparoscopic radical prostatectomy for a single experienced surgeon: comparison with simultaneous laparoscopic radical prostatectomy. The world journal of men's health. 2015;33:30-35.

16. Wagenhoffer R, Gruner M, Schymik J, et al. Switching from endoscopic extraperitoneal radical prostatectomy to robot-assisted laparoscopic prostatectomy: comparing outcomes and complications. Urol Int. 2015;95:380-385.

17. Pearce SM, Pariser JJ, Patel SG, Anderson BB, Eggener SE, Zagaja GP. The impact of days off between cases on perioperative outcomes for robotic-assisted laparoscopic prostatectomy. World journal of urology. 2015:1-6.

18. Lee JY, Mucksavage P, Sundaram CP, McDougall EM. Best practices for robotic surgery training and credentialing. The Journal of urology. 2011;185:1191-1197.

19. Lovegrove C, Novara G, Mottrie A, et al. Structured and modular training pathway for robot-assisted radical prostatectomy (RARP): validation of the RARP assessment score and learning curve assessment. European Urology. 2015. 
Figures and Tables

Table 1. Baseline characteristics of study participants.

\begin{tabular}{|l|l|l|l|l|l|}
\hline Property & Q1 & Q2 & Q3 & Q4 & Overall \\
\hline Case number & $1-100$ & $101-200$ & $201-300$ & $301-400$ & $1-400$ \\
\hline Start & $3 / 2012$ & $4 / 2013$ & $12 / 2013$ & $9 / 2014$ & \\
\hline Days & 384 & 249 & 283 & 210 & 1126 \\
\hline Cases/week & 1.8 & 2.8 & 2.5 & 3.3 & 2.6 \\
\hline Median age & 63.1 & 64.4 & 63.4 & 64.2 & 63.8 \\
\hline Median ASA & 3 & 2 & 3 & 3 & 3 \\
\hline Median BMI & 28.7 & 28.1 & 30.0 & 30.0 & 29.4 \\
\hline Median gland volume & 32.0 & 33.5 & 35.0 & 34.5 & 34.0 \\
\hline Mean cores positive & 4.8 & 2.8 & 3.3 & 3.3 & 3.6 \\
\hline Median PSA & 6.9 & 6.9 & 7.7 & 6.5 & 6.9 \\
\hline
\end{tabular}

No statistically significant differences between quartile were noted. ASA: American Society of Anesthesiologists Score; BMI: body mass index: PSA: prostate-specific antigen.

\section{Table 2. Operative characteristics of study participants}

\begin{tabular}{|c|c|c|c|c|c|}
\hline Property & Q1 & Q2 & Q3 & Q4 & Overal \\
\hline $\begin{array}{l}\text { Mean OR } \\
\text { time }\end{array}$ & $207.4^{*}$ & 184.4 & 177.6 & 179.2 & 187.2 \\
\hline $\begin{array}{l}\text { Mean } \\
\text { Estimated } \\
\text { blood loss }\end{array}$ & $255.1^{*}$ & 246.4 & 248.6 & 213.6 & 240.9 \\
\hline $\begin{array}{l}\text { Number of } \\
\text { transfusions }\end{array}$ & 1 & 0 & 1 & 1 & 1 \\
\hline $\begin{array}{l}\text { No nerve- } \\
\text { sparing }\end{array}$ & 23 & 28 & 39 & 24 & 28.5 \\
\hline $\begin{array}{l}\text { Unilateral } \\
\text { nerve-sparing }\end{array}$ & 13 & 9 & 6 & 6 & 8.5 \\
\hline $\begin{array}{l}\text { Bilateral } \\
\text { nerve-sparing }\end{array}$ & 64 & 63 & 50 & 64 & 60.3 \\
\hline $\begin{array}{l}\text { Length of } \\
\text { stay }\end{array}$ & 2 & 2 & 2 & 2 & 2 \\
\hline
\end{tabular}

*Statistically significant difference relative to most recent quartile (Q4). OR: operating room. 


\begin{tabular}{|l|l|l|l|l|l|}
\hline \multicolumn{6}{|l|}{ Table 3. Pathologic characteristics of study participants } \\
\hline Property & Q1 & Q2 & Q3 & Q4 & Overall \\
\hline $\begin{array}{l}\text { Number } \\
\text { Gleason 6 }\end{array}$ & 23 & 19 & 19 & 15 & 19 \\
\hline $\begin{array}{l}\text { Number } \\
\text { Gleason 7 }\end{array}$ & 67 & 70 & 65 & 68 & 67.5 \\
\hline $\begin{array}{l}\text { Number } \\
\text { Gleason 8-10 }\end{array}$ & 7 & 9 & 11 & 12 & 9.8 \\
\hline Number ECE & 39 & 29 & 44 & 41 & 38.3 \\
\hline Number SVI & 11 & 8 & 17 & 10 & 11.5 \\
\hline N1 disease & 4 & 2 & 4 & 2 & 3 \\
\hline $\begin{array}{l}\text { Prostate } \\
\text { weight (g) }\end{array}$ & 48.4 & 52.8 & 52.5 & 55.1 & 52.2 \\
\hline $\begin{array}{l}\text { Positive } \\
\text { margins }\end{array}$ & 29 & 15 & 19 & 19 & 20.5 \\
\hline $\begin{array}{l}\text { T3 positive } \\
\text { margins }\end{array}$ & 17 & 8 & 16 & 12 & 13.3 \\
\hline $\begin{array}{l}\text { T2 positive } \\
\text { margins }\end{array}$ & 12 & 7 & 3 & 7 & 7.3 \\
\hline $\begin{array}{l}\text { Apical } \\
\text { margins }\end{array}$ & 18 & 10 & 8 & 7 & 10.8 \\
\hline N0 & & 16 & 7 & \\
\hline
\end{tabular}

No statistically significant differences were noted between quartiles. ECE: extracapsular extension; SVI: seminal vesicle invasion.

Table 4. Overall, T3 and T2 margin rates compared between quartile 1 and the most recent
quartile (Q4)
\begin{tabular}{|l|l|l|l|}
\hline Property & Overall & T3 & T2 \\
\hline Q1 Positive margins & 29 & 17 & 12 \\
\hline Q4 Positive margins & 19 & 12 & 7 \\
\hline Odds ratio & 1.74 & 1.45 & 1.69 \\
\hline $95 \%$ confidence interval & $0.90-3.40$ & $0.62-3.40$ & $0.62-4.60$ \\
\hline p & 0.1 & 0.3 & 0.3 \\
\hline
\end{tabular}




\begin{tabular}{|c|c|c|}
\hline Author & Previous experience & Reported learning curve \\
\hline Jaffe et al $2009^{10}$ & $\begin{array}{l}3091 \text { LRP ( } 535 \text { before } \\
\text { RARP era), unspecified } \\
\text { number of surgeons }\end{array}$ & $\begin{array}{l}189 \text { cases for OR time and PSM } \\
\text { rate }\end{array}$ \\
\hline $\begin{array}{l}\text { Wolanski et al } \\
2012^{11}\end{array}$ & 200-300 each (2 surgeons) & $\begin{array}{l}\text { None detected in T2 or T3 PSM } \\
\text { rate, 3-month PSA recurrence, } \\
\text { OR time - } 20 \text { cases }\end{array}$ \\
\hline Barret et al. $2011^{12}$ & >300 LRPs (2 surgeons) & 100 for pT2 PSM \\
\hline $\begin{array}{l}\text { Stolzenburg et al } \\
2013^{13}\end{array}$ & $\begin{array}{l}1000 \text { LRP each (2 } \\
\text { surgeons) }\end{array}$ & $\begin{array}{l}\text { Not detected for PSM rate or 3- } \\
\text { month detectable PSA value } \\
\text { EBL and OR time - not reached in } \\
100 \text { cases }\end{array}$ \\
\hline $\begin{array}{l}\text { Di Pierro et al } \\
2014^{14}\end{array}$ & $>50$ LRP (1 surgeon) & $\begin{array}{l}\text { PSM rate not reported. } 175 \text { cases } \\
\text { for complication rate. OR time } \\
\text { declined after first } 59 \text { cases. }\end{array}$ \\
\hline Ku \& Ha $2015^{15}$ & 369 LRP (1 surgeon) & $\begin{array}{l}\text { None detected T2 PSM rate, } 3 \text { - } \\
\text { month intercourse or pad use rate } \\
\text { OR time }-20 \text { cases }\end{array}$ \\
\hline $\begin{array}{l}\text { Wagenhoffer et al } \\
2015^{16}\end{array}$ & 86 LRP (1 surgeon) & $\begin{array}{l}\text { Not detected for PSM rate. } \\
\text { Increased OR time compared to } \\
\text { LRP in first } 100 \text { cases. Less blood } \\
\text { loss in first } 100 \text { cases. }\end{array}$ \\
\hline
\end{tabular}

LRP: laparoscopic radical prostatectomy; RARP: robotically assisted laparoscopic radical prostatectomy. 